\title{
X-ray microscopy techniques for nanostructure analysis.
}

\author{
Janos Kirz
}

Advanced Light Source, Lawrence Berkeley National Laboratory, Berkeley, CA 94720, and Department of Physics and Astronomy, Stony Brook University, Stony Brook, NY 11794-3800

X-rays have been used for a long time to characterize nanostructures. Shapes and sizes of nanoparticles are routinely determined by small and large angle X-ray scattering. Elemental and chemical information is gained from X-ray fluorescence and absorption spectroscopy, respectively. These techniques are useful to study ensemble averages of macroscopic quantities of the specimen. Recent advances are starting to make it possible to use X-ray microscopy to study clusters, or even individual nanoparticles in isolation, or as parts of a larger system.

A major advantage of using X-rays is the penetrating power of the radiation. The specimen may be relatively thick (e.g. nanoparticles embedded in a matrix, or a complex structure made up of nanoparticles). In addition, fluorescence and absorption spectra are beginning to be collected from individual nanostructures, extending the elemental and chemical analysis capabilities to the nanoworld.

Several approaches to X-ray microscopy of nanostructures are under development. [1]

- Zone plates are used to form nanoprobes for scanning microscopy and spectromicroscopy $[2]$.

- Other forms of X-ray optics, such as Kirkpatrick-Baez mirror systems are rapidly improving in resolution, and offer broad spectral tunability in microprobe applications [1].

- Zone plates are also used as objective lenses in full-field microscopes, and the resolution in this form of imaging has already reached the $15 \mathrm{~nm}$ level [3].

- The technique of Diffraction Microscopy, where the diffraction pattern of a non-crystalline specimen is recorded, and the object is reconstructed by an iterative algorithm [4] dispenses with X-ray optics altogether.

The ultimate limitation to the finest spatial resolution one can obtain in X-ray microscopy is either radiation damage [5] or, in the most radiation-hard specimens, it is the wavelength of the X-rays. Where multiple identical copies of the specimen can be obtained, and arrayed in a regular structure as in a crystal, the radiation dose can be shared among the many repeats, and atomic resolution is possible, as demonstrated by crystallographers on a routine basis. But what if the identical objects refuse to crystallize? With a suitably powerful X-ray laser with femtosecond pulse duration, one should still be able to exceed the radiation damage limit, by recording the diffraction pattern before the nanoparticle or molecule explodes. [6] Such a laser, the LCLS, is under construction at the Stanford Linear Accelerator Center [7]. If the diffraction pattern from a single object is not strong enough to allow reconstruction at the desired resolution, a stream of identical objects may be used. In this case the diffraction patterns need to be sorted depending on the orientation of the specimen, much as in electron-cryo-tomography [8]. Alternatively one may consider ways of pre-aligning the identical specimens to simplify the analysis. [9]. 
Related to this last scheme is the suggestion by Spence and Doak [10]. They point out that if a large collection of identical, non-crystallizable specimens is available these may be aligned using the field of an intense infrared laser. It may then be possible to do "serial crystallography" [10], where the radiation dose to any one specimen is small enough that the diffraction pattern may be collected using a conventional synchrotron X-ray beam as the laser-aligned nanostructures or macromolecules stream by. The first tests of this idea are scheduled for the near future.

Not only is X-ray microscopy suitable for the characterization of nanostructures, nanoparticles are also used as labels or markers in X-ray microscopy and tomography. Nanodots made of heavy metals are particularly useful, since these are easily identified, and can be functionalized to label structures of interest. [11], [12]

References

[1] Y. Kagoshima, Proceedings of the 8th International Conference on X-ray Microscopy, IPAP Conference Series 7, Institute of Pure and Applied Physics, Tokyo, (2006).

[2] J. Maser, et al., Microsc. Microanal. 11 (Suppl. 2) (2005) 680.

[3] W. Chao et al., Nature, 435 (2005) 1210.

[4] J. Miao et al., Nature, 400 (1999) 342.

[5] M. Howells et al., Electr. Spectr. Rel. Phenom. (2006) submitted

[6] R. Neutze et al., Nature, 406 (2000) 752.

[7] see http://www-ssrl.slac.stanford.edu/lcls/

[8] J. Frank, Electron Tomography: Three-Dimensional Imaging with the Transmission Electron Microscope, Springer, Berlin, 1992

[9] H. Chapman et al., Report on the Instrument Development Workshop for Biological Imaging Experiments at LCLS, Lawrence Livermore National Laboratory Report UCRL-PROC-206061 (2004) http://www.llnl.gov/tid/lof/documents/pdf/310930.pdf

[10] J. C. H. Spence and R. B. Doak, Phys. Rev. Lett. 92 (2004) 198102.

[11] C. A. Larabell and M. A. Le Gros. Mol. Biol. Cell, 15 (2004) 957.

[12] Supported by the Office of Basic Energy Sciences, US Department of Energy. 